\title{
Effect of Birth Weight, Gestational Age, Sex and Intrauterine Growth on Mortality and Morbidity Profile of very Low Birth Weight Babies (VLBW)
}

\author{
Rabindran ${ }^{1}$, Parakh $\mathbf{H}^{2}$, Ramesh $\mathrm{J} \mathrm{K}^{3}$, Reddy $\mathbf{P}^{4}$ \\ ${ }^{1}$ Dr. Rabindran, Junior Consultant Neonatologist, ${ }^{2}$ Dr.Hemant Parakh, Consultant Neonatologist, ${ }^{3}$ Dr.Ramesh J K, Consultant \\ Pediatrician, ${ }^{4}$ Dr.Prashant Reddy, Consultant Pediatrician, Sunrise Superspeciality Children's Hospital. Hyderabad, AP, India
}

Address for correspondence: Dr Rabindran, E mail: rabindranindia@yahoo.co.in

\begin{abstract}
Introduction: Very Low birth weight is associated with serious neonatal morbidity. Biologic factors are major determinants in their outcome. We analysed the effect of birth-weight, gestation, sex and intrauterine growth in the mortality and morbidity profile of VLBW babies during the neonatal period. Methodology: This is a cross sectional retrospective observational study from April-2012 to August-2014. Baseline demographics, disease features of 97 VLBW babies were analyzed. Results: Survival at discharge was $91.75 \%$. There was significant difference in need of ventilation, surfactant, Apnea, ROP, IVH $>/=$ Grade-II, Culture-negative Sepsis among all gestational subgroups. Survival increased as gestation advanced. Maximum decrease in mortality has occurred beyond 28 weeks. Maximum odds difference in need of ventilation, BPD was noted around 28 weeks. Major difference in HS-PDA, IVH, NEC were noted around 30 weeks. Significant difference in need of surfactant, apnea and anemia was observed around 32 weeks. Major decrease in HMD, Hyperbirubinemia and sepsis were identified around 34 weeks. Analysing intrauterine growth, Significant difference in Need of ventilation, surfactant use, IVH, NEC, Anemia and death was noted between AGA and SGA. Analysing birth weight wise, Survival improved as birth-weight increased. There was significant difference in HS-PDA and IVH in all birth-weight subgroups. Maximum decrease in death was noted in babies $>1000 \mathrm{~g}$. Maximum odds difference in BPD, Apnea, Hyperbilirubinemia, IVH, Anemia, ROP and culture negative sepsis occured around 800g. Major difference in HMD, NEC, Culture positive sepsis was observed around $1000 \mathrm{~g}$. Significant odds difference in HS-PDA occurred around 1200g. Maximum decrease in need of surfactant and ventilation was noted around $1400 \mathrm{~g}$. Conclusion: There was no difference between male and female in survival or morbidities. Survival improved with advancing Gestation, Intrauterine growth and Birth-weight. Analysing intrauterine growth, Significant difference in Need of ventilation, surfactant, IVH, NEC, Anemia and death was noted between AGA and SGA. Significant variations in morbidity profile were noted among birth weight and gestational age subgroups.
\end{abstract}

Keywords: Survival, Very low birth weight, gestational age, Intrauterine growth retardation

\section{Introduction}

The very low birth weight babies are at risk from a wide range of hazards resulting from immaturity of structure and function of various organs [1]. With advanced perinatal \& neonatal care, survival of VLBW infants has increased globally. Well equipped, experienced NICUs also have contributed to the increasing survival of VLBW neonates $[2,3,4,5]$. Studies have reported normal outcomes in $73 \%$ of these babies figures vary widely from country to country with reports of up to $90 \%$ survival from developed countries to $40 \%$ in the developing world [6]. According to 2010 National Vital Statistics Report, in 2006 the mortality rate among infants with VLBW was Manuscript received: $15^{\text {th }}$ Oct 2014

Reviewed: $26^{\text {th }}$ Oct 2014

Author Corrected; $29^{\text {th }}$ Oct 2014

Accepted for Publication: $15^{\text {th }}$ Nov 2014
240.4 per 1000 live births [7]. There is paucity of data regarding outcome of VLBW babies based on gestational age, birth weight applicable to our population. Outcomes in VLBW infants are best understood as an interaction between biological vulnerability \& environmental factors. Does biological factors (birth weight, gestational age, sex, intrauterine growth) has a significant impact on survival and morbidity profile during neonatal period is our research question.

Primary Outcome: To determine the effect of gestational age, birth weight, sex and intrauterine growth in the mortality and morbidity profile of very low birth weight babies during the neonatal period. 
Secondary Outcome: To analyse the morbidities like Respiratory Distress Syndrome (RDS/HMD), surfactant usage, Bronchopulmonary Dysplasia (BPD), Apnea, Hemodynamically significant Patent Ductus Arteriosus (HS-PDA) requiring treatment, Necrotising Enterocolitis (NEC), Intraventricular Hemorrhage (IVH) $>/=$ Grade II, Anemia requiring PRBC transfusion, Sepsis (both culture positive and culture negative Sepsis) and Retinopathy of Prematurity (ROP).

Study Period: 2 years and 5months (April 2012 to August 2014).

Study Design: Cross sectional Retrospective observational study.

Inclusion Criteria: All babies with birth weight less than $1.5 \mathrm{~kg}$, admitted at Sunrise Superspeciality Children's Hospital were included in the study.

Methodology: $\mathrm{T}$ his cross- sectional retrospective study was performed from April 2012 to August 2014 on all hospitalized VLBW babies. Relevant pre and perinatal data upto the time of discharge or death , including complications during the course of hospitalization, were collected from the case notes, documented on a predesigned proforma with preformed diagnostic criteria and analysed. The gestational ages were determined by obstetric assessment and modified Ballards score, when antenatal reports were not available [8]. Hyaline membrane disease was diagnosed according to clinical and radiological findings. Retinopathy of prematurity was diagnosed by an ophthalmologist and classified in grades 1 to 5 according to international classification [9]. BPD was diagnosed according to the criteria of Bancalari et al [10] including clinical and radiographic features together with the requirement of oxygen therapy at 28 days of age. Bronchopulmonary dysplasia (BPD) was defined by oxygen requirements at 28 days of life and chronic radiographic changes [11]. The diagnosis of sepsis was confirmed by isolation of the organism in the blood. Patent ductus arteriosus was diagnosed clinically and confirmed by echocardiography. The diagnosis of Intraventricular Hemorrhage was made by ultrasonogram and was classified according to Papile and Bursten [12]. NEC was determined by the clinical and radiological criteria of Bell et al [13] and only definite NEC (Bell stages II-III) was included. Growth was plotted in Fenton growth charts. Interventions: Preterm care as per standard unit protocol. Data Analysis: Detailed information including gestational age at diagnosis, birth weight were collected from hospital records of all VLBW admissions. Outcomes were classified as neonatal survival or death. Categorical variables were analyzed using Chisquare analysis with Yates correction. Student't' test was used to compare the means. A p-value of $<0.05$ was considered significant.

\section{Results}

There were total 1191 newborn admissions during the study period. Among these 1191 babies, 97 babies $(8.14 \%)$ were very low birth weight babies and were included in the study. Survival rate at discharge among VLBW babies was $91.75 \%$. Among VLBW babies $55.67 \%(n=54)$ were male \& $44.32 \%(n=43)$ were female. However there was no statistical significance (2-Tailed probability $=0.2633$ ). Analysing gestational age distribution, $24.74 \%(n=24) \quad$ were $31-32$ weeks, $20.61 \%(n=20)$ were $29-30$ weeks and $18.55 \%(n=18)$ were 33-34 weeks of gestation. For statistical analysis, birth weight was divided in to 5 subgroups. 7 babies were $<800$ g, 10 babies between 800 to 1000 g, 31 babies between 1000 to $1200 \mathrm{~g}, 24$ babies between 1200 to 1400 $\mathrm{g}$ and 25 babies were between 1400 to $1500 \mathrm{~g}$. When birth weights were plotted against the gestational age on Fenton growth charts, $56.7 \%(\mathrm{n}=55)$ were AGA, $43.29 \%(\mathrm{n}$ $=42$ ) were SGA. However 2 tailed probability $=0.1857$ was not significant. For statistical analysis, gestational age was divided in to 5 subgroups. 10 babies were $<28$ weeks, 21 babies between 28 to 30weeks, 34 babies between 30 to 32 weeks, 25 babies between 32 to 34 weeks and 7 babies were $>34$ weeks.

\section{Effect of Gestational Age on Mortality \& Morbidity Profile of VLBW Babies}

Analysing the gestational subgroups, we estimated the morbidity and mortality between the subgroups. 
Figure: 1

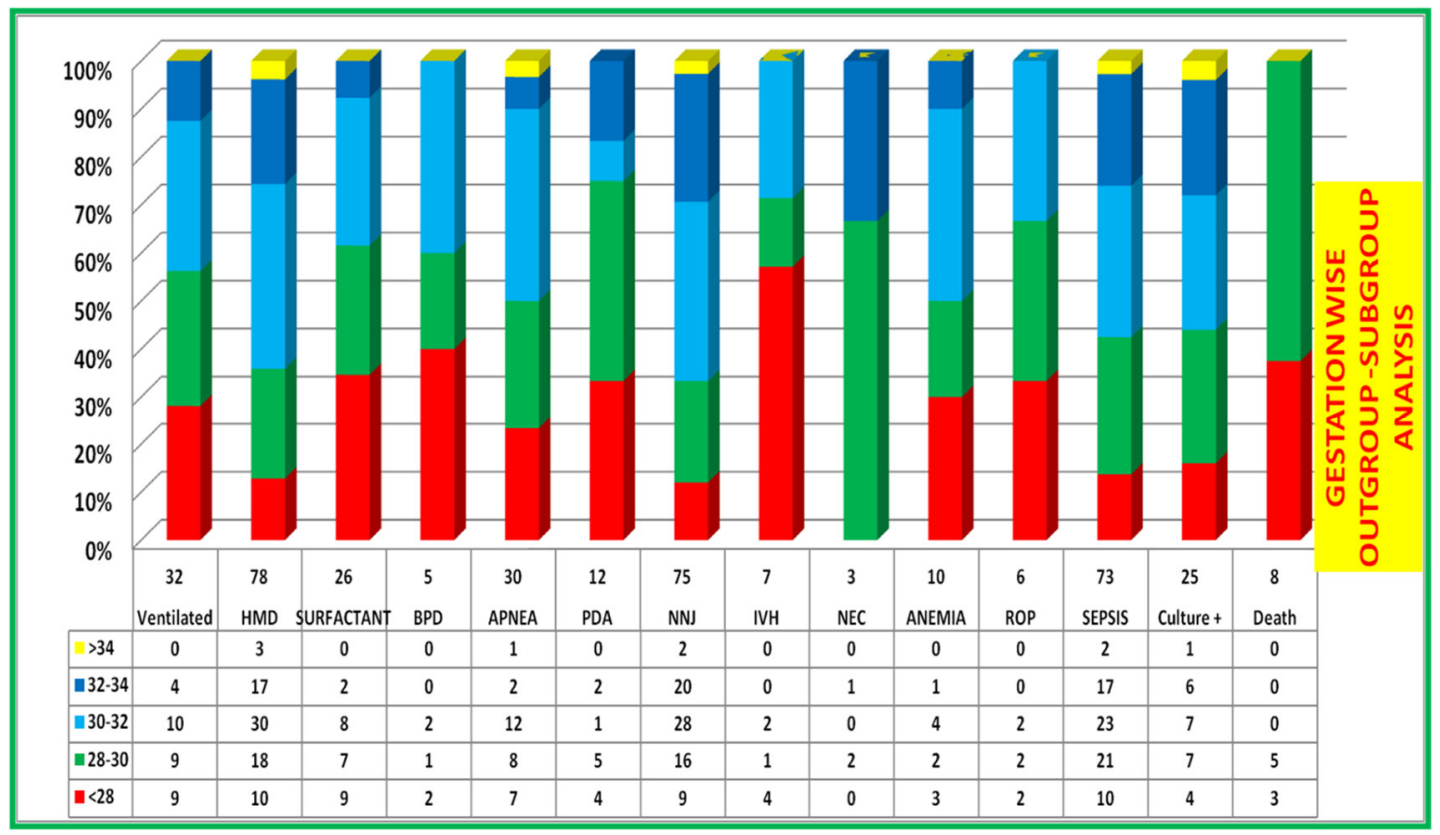

Figure 1 shows the outcome of VLBW babies based upon the gestational subgroups.

Table1: Distribution of Morbidity Profile-Gestation Wise-Subgroup Analysis

\begin{tabular}{|c|c|c|c|c|c|c|c|c|c|c|c|c|}
\hline & $<28$ & $>28$ & $\mathbf{p}$ & $<30$ & $>30$ & $\mathbf{p}$ & $<32$ & $>32$ & $\mathbf{p}$ & $<34$ & $>34$ & $\mathbf{p}$ \\
\hline Ventilated $\%$ & 90 & 26.43 & $<0.00001$ & 58.06 & 21.21 & $<0.00001$ & 41.17 & 12.5 & 0.0001 & 35.55 & $\mathbf{0}$ & $<0.00001$ \\
\hline Hmd \% & 100 & 78.16 & 0.1217 & 90.32 & 75.75 & 0.2652 & 89.23 & 62.5 & 0.0310 & 83.33 & 42.85 & 0.0002 \\
\hline Surfactan $\%$ & 90 & 19.54 & $<0.00001$ & 51.61 & 15.15 & $<0.00001$ & 36.92 & 6.25 & $<0.00001$ & 28.88 & $\mathbf{0}$ & $<0.00001$ \\
\hline Bpd \% & 20 & 3.44 & 0.0005 & 9.67 & 3.03 & 0.0648 & 7.69 & $\mathbf{0}$ & $<0.00001$ & 5.55 & $\mathbf{0}$ & $<0.00001$ \\
\hline Apnea $\%$ & 70 & 26.43 & $<0.00001$ & 48.38 & 22.72 & 0.0022 & 41.53 & 9.37 & $<0.00001$ & 32.22 & 14.28 & 0.0085 \\
\hline Pda $\%$ & 40 & 9.19 & $<0.00001$ & 29.03 & 4.54 & $<0.0001$ & 15.38 & 6.25 & 0.0515 & 12.22 & 14.28 & $<0.0001$ \\
\hline Nnj \% & 90 & 75.86 & 0.2794 & 80.64 & 75.75 & 0.7008 & 81.53 & 68.75 & 0.3045 & 81.11 & 28.57 & $<0.0001$ \\
\hline Ivh \% & 40 & 3.44 & $<0.00001$ & 16.12 & 3.03 & 0.0026 & 10.76 & $\mathbf{0}$ & $<0.00001$ & 7.77 & $\mathbf{0}$ & $<0.00001$ \\
\hline Nec $\%$ & $\mathbf{0}$ & 3.44 & $<0.00001$ & 6.45 & 1.51 & 0.083 & 3.07 & 3.12 & 0.9842 & 3.33 & $\mathbf{0}$ & $<0.00001$ \\
\hline Anemia \% & 30 & 8.04 & 0.0003 & 16.12 & 7.57 & 0.0820 & 13.84 & 3.12 & 0.0093 & 11.11 & 0 & $<0.00001$ \\
\hline Rop \% & 20 & 4.59 & 0.0017 & 12.9 & 3.03 & 0.0136 & 9.23 & $\mathbf{0}$ & $<0.00001$ & 6.66 & $\mathbf{0}$ & $<0.00001$ \\
\hline Sepsis\% & 100 & 72.41 & 0.0438 & 100 & 63.63 & 0.0055 & 83 & 59.37 & 0.0494 & 78.88 & 28.57 & $<0.00001$ \\
\hline Culture $+\%$ & 40 & 24.13 & 0.0493 & 35.48 & 21.21 & 0.0603 & 27.69 & 21.87 & 0.4159 & 26.66 & 14.28 & 0.055 \\
\hline Death\% & 30 & 5.74 & $<0.00001$ & 25.8 & $\mathbf{0}$ & $<0.00001$ & 7.69 & $\mathbf{o}$ & $<0.00001$ & 8.88 & $\boldsymbol{O}$ & $<0.00001$ \\
\hline
\end{tabular}

Table 1 shows the distribution of morbidity profile based upon the gestational subgroups.

A one-sample t-test between proportions was performed to determine whether there was a significant difference among morbidities and death based on gestational age subgroups. The t-statistic was analysed and $\mathrm{p}$ value $<0.05$ was considered significant. Gestation wise $<28 \&>28$ weeks; $<30 \&>30$ weeks; $<32 \&>32$ weeks; $<34 \&>34$ weeks were analysed. It is well known that immature infants (particularly those born $<32$ weeks ) are at higher risk for mortality and morbidity and failure to consider gestational age leads to major problem in interpretation that hinder decision-making at both clinical and public health levels [17]. There was significant difference in need of ventilation and surfactant use, Apnea, ROP, (IVH) $>/=$ Grade II, Culture negative Sepsis in all subgroups. However culture positive was more significant between $<28 \&>28$ weeks. Significant difference in Jaundice was noted between $<34 \&>34$ weeks. Significant difference in HMD was noted between $<$ $32 \&>32$ weeks and $<34 \&>34$ weeks. BPD and anemia requiring PRBC transfusion, was significant in all subgroups except $<30 \&>30$ weeks. Hemodynamically significant Patent Ductus Arteriosus (HS-PDA) requiring treatment, was significant in all subgroups except $<32 \&>32$ weeks. NEC was significant between $<28 \&>28$ weeks and $<34 \&>34$ weeks. Statistical significant difference in death was noted in all subgroups. Survival increased significantly as gestation advanced. 
Table 2: Comparison of Proportions / Odds among Gestational Subgroups

\begin{tabular}{|c|c|c|c|c|}
\hline Morbidity & $</>28$ & $</>30$ & $</>32$ & $</>34$ \\
\hline Ventilated & 3.4 & 2.73 & 3.29 & $>$ \\
\hline HMD & 1.27 & 1.19 & 1.42 & 1.94 \\
\hline Surfactant & 4.6 & 3.4 & 5.9 & $>$ \\
\hline BPD & 5.81 & 3.19 & $>$ & $>$ \\
\hline Apnea & 2.64 & 2.12 & 4.43 & 2.25 \\
\hline PDA & 4.35 & 6.39 & 2.46 & $>$ \\
\hline NNJ & 1.18 & 1.06 & 1.18 & 2.83 \\
\hline IVH & 11.6 & 5.32 & $>$ & $>$ \\
\hline NEC & $\mathbf{0}$ & 4.27 & 0.98 & $>$ \\
\hline Anemia & 3.73 & 2.12 & 4.43 & $>$ \\
\hline ROP & 4.35 & 4.25 & $>$ & $>$ \\
\hline sepsis & 1.38 & 1.57 & 1.39 & 2.76 \\
\hline Culture+ & 1.65 & 1.67 & 1.26 & 1.86 \\
\hline DEATH & 5.22 & $>$ & $>$ & $>$ \\
\hline
\end{tabular}

Table 2 depicts the comparison of proportions based upon gestational subgroups.

Analysing the proportions of morbidities and mortality among each gestational subgroup, we compared the ratio of morbidities and mortality in each subgroup to

1. estimate maximum risk difference of morbidity and mortality

2. to analyse the gestational age when 0 mortality and 0 individual morbidity was attained.

No death was noted beyond 30 weeks. No BPD, IVH >/= Grade II \& ROP were noted beyond 32 weeks. No need of assisted ventilation, surfactant need, Hemodynamically significant Patent Ductus Arteriosus, NEC \& Anemia requiring transfusion were noted beyond 34 weeks. Maximum difference in death, need of assisted ventilation \& BPD were noted around 28 weeks. Maximum difference in Hemodynamically significant Patent Ductus Arteriosus, IVH $>/=$ Grade II \& NEC were noted around 30 weeks. Maximum difference in surfactant usage, apnea and anemia were noted around 32 weeks. Maximum difference in the occurrence of HMD, Hyperbilirubinemia and sepsis were noted around 34 weeks.

\section{Effect of sex in mortality \& morbidity profile of VLBW babies}


Figure: 2- Sex wise distribution of morbidity profile-subgroup analysis

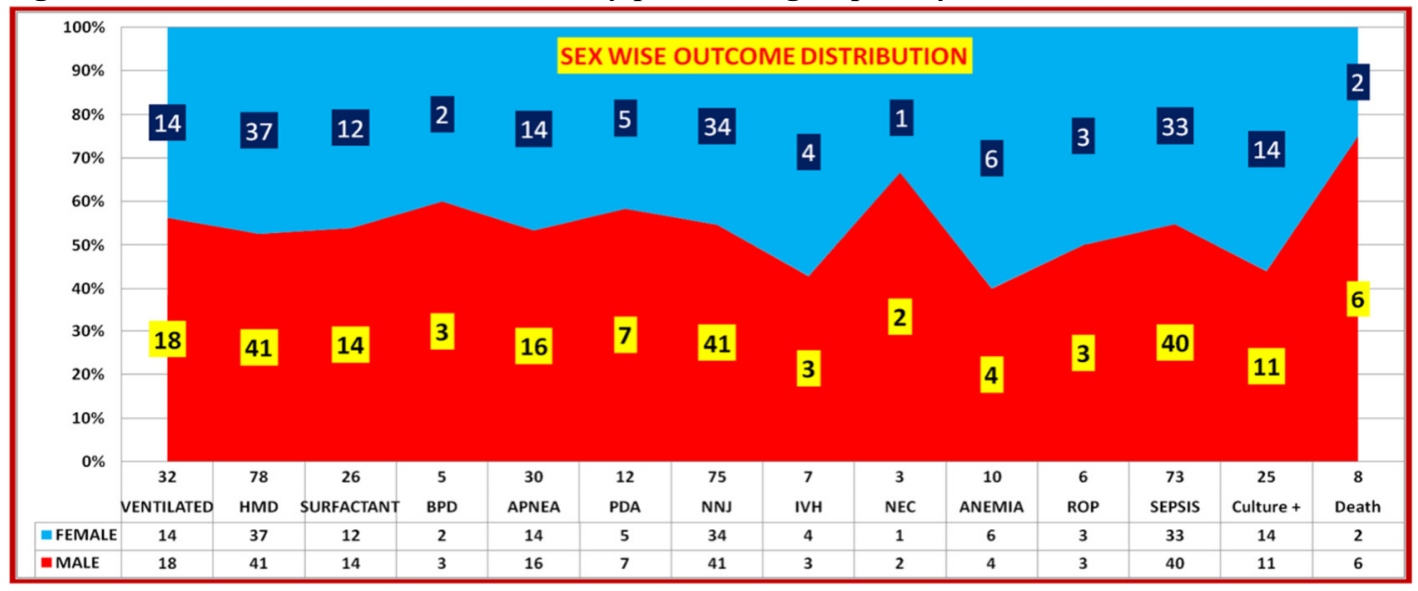

Table 3: Sex Wise Distribution Of Morbidity Profile-Subgroup Analysis

\begin{tabular}{|l|c|c|c|}
\hline VENTILATED \% & MALE & FEMALE & p \\
\hline HMD \% & 33.33 & 32.55 & 0.9248 \\
\hline SURFACTANT \% & 75.92 & 86.04 & 0.4340 \\
\hline BPD \% & 25.92 & 27.9 & 0.7909 \\
\hline APNEA \% & 5.55 & 4.65 & 0.7819 \\
\hline PDA\% & 29.62 & 32.55 & 0.7150 \\
\hline NNJ \% & 12.96 & 11.62 & 0.7906 \\
\hline IVH \% & 75.92 & 79.06 & 0.8043 \\
\hline NEC \% & 5.55 & 9.30 & 0.3380 \\
\hline ANEMIA \% & 3.7 & 2.32 & 0.5803 \\
\hline ROP \% & 7.4 & 13.95 & 0.1617 \\
\hline SEPSIS\% & 5.55 & 6.97 & 0.6933 \\
\hline CULTURE POSITIVE\% & 74.07 & 76.74 & 0.8309 \\
\hline DEATH\% & 20.37 & 32.55 & 0.0977 \\
\hline
\end{tabular}

Table 3 shows the sex wise comparison of morbidity profile of VLBW babies.

There was no Statistical significant difference in death and morbidities between male and female subgroups.

\section{Effect of Intrauterine Growth in Mortality \& Morbidity Profile of VLBW Babies}


Figure 3: Intrauterine Growth Wise Distribution of Morbidity Profile-Subgroup Analysis

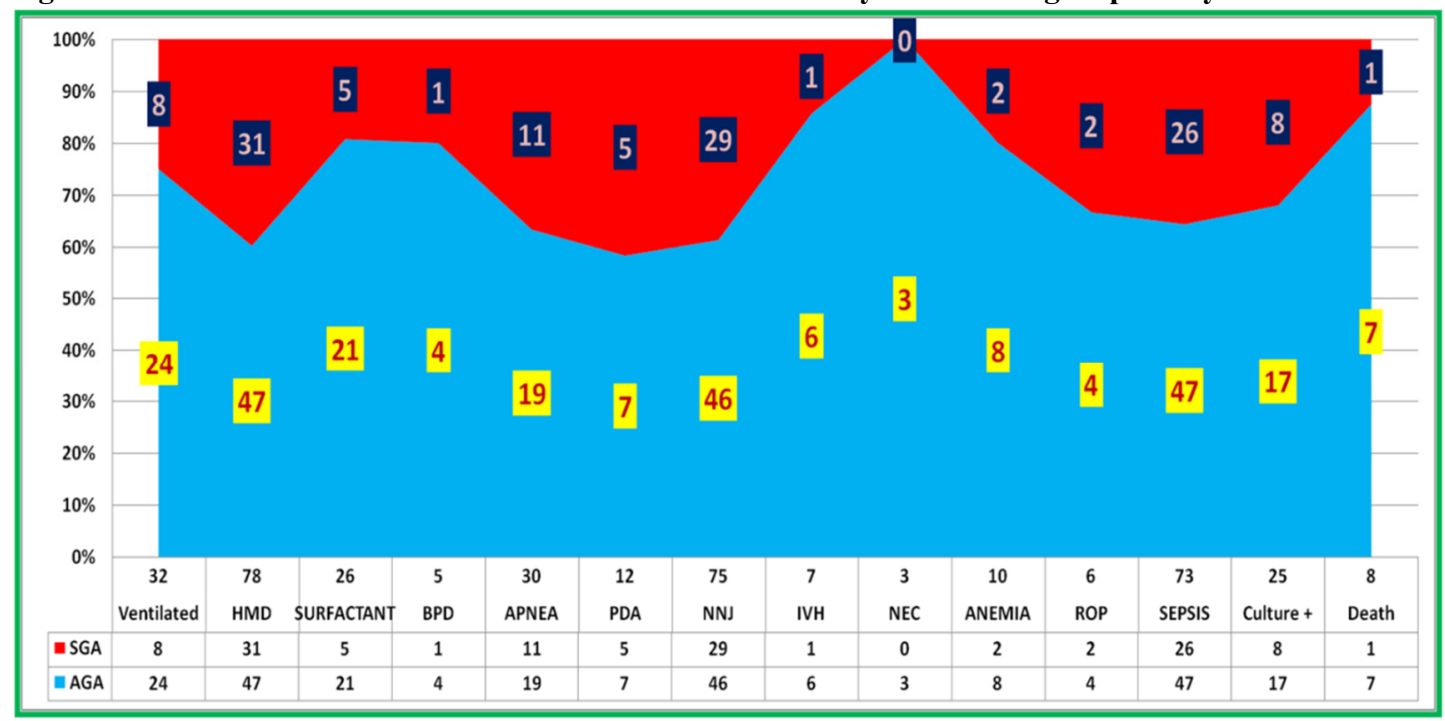

Figure 3 shows the outcome of VLBW babies based upon intrauterine growth.

Table 4 : Growth Wise Distribution of Morbidity Profile- Subgroup Analysis

\begin{tabular}{|c|c|c|c|}
\hline & AGA & SGA & p \\
\hline VENTHLATED \% & 43.63 & 19.04 & $\mathbf{0 . 0 0 1 8}$ \\
\hline HIMD \% & 85.45 & 73.8 & $\mathbf{0 . 3 6 3 5}$ \\
\hline SURFACTANT \% & 38.18 & 11.9 & 0.0002 \\
\hline BPD \% & 7.27 & 2.38 & $\mathbf{0 . 1 1 9 7}$ \\
\hline APNEA \% & 34.54 & 26.19 & $\mathbf{0 . 2 9 1 2}$ \\
\hline PDA \% & 12.72 & 11.9 & $\mathbf{0 . 8 7 1 0}$ \\
\hline NNJ \% & 83.63 & 69.04 & $\mathbf{0 . 2 4 4 4}$ \\
\hline IVH \% & 10.9 & 2.38 & $\mathbf{0 . 0 1 9 9}$ \\
\hline NEC \% & 5.45 & 0 & $<\mathbf{0 . 0 0 0 1}$ \\
\hline ANEMIA \% & 14.54 & 4.76 & $\mathbf{0 . 0 2 6 8}$ \\
\hline ROP \% & 7.27 & 4.76 & $\mathbf{0 . 4 7 6 6}$ \\
\hline SEPSIS \% & $\mathbf{8 5 . 4 5}$ & 61.9 & $\mathbf{0 . 0 5 4 4}$ \\
\hline CULTURE + \% & 30.90 & 19.04 & $\mathbf{0 . 0 9 6 9}$ \\
\hline DEATH \% & 12.72 & 2.38 & $\mathbf{0 . 0 0 7 8}$ \\
\hline
\end{tabular}

Table 4 shows the intrauterine growth wise comparison of morbidity profile of VLBW babies.

There was significant difference in Need of ventilation, surfactant use, IVH, NEC, Anemia and death was noted between AGA and SGA.

\section{Effect of Birth Weight in Mortality \& Morbidity Profile of VLBW Babies}

Analysing the Birth Weight subgroups, we estimated the morbidity and mortality between the subgroups. We analysed the variation of mortality and morbidity among, 
Figure 4 : Birth Weight Wise Distribution Of Morbidity Profile-Subgroup Analysis

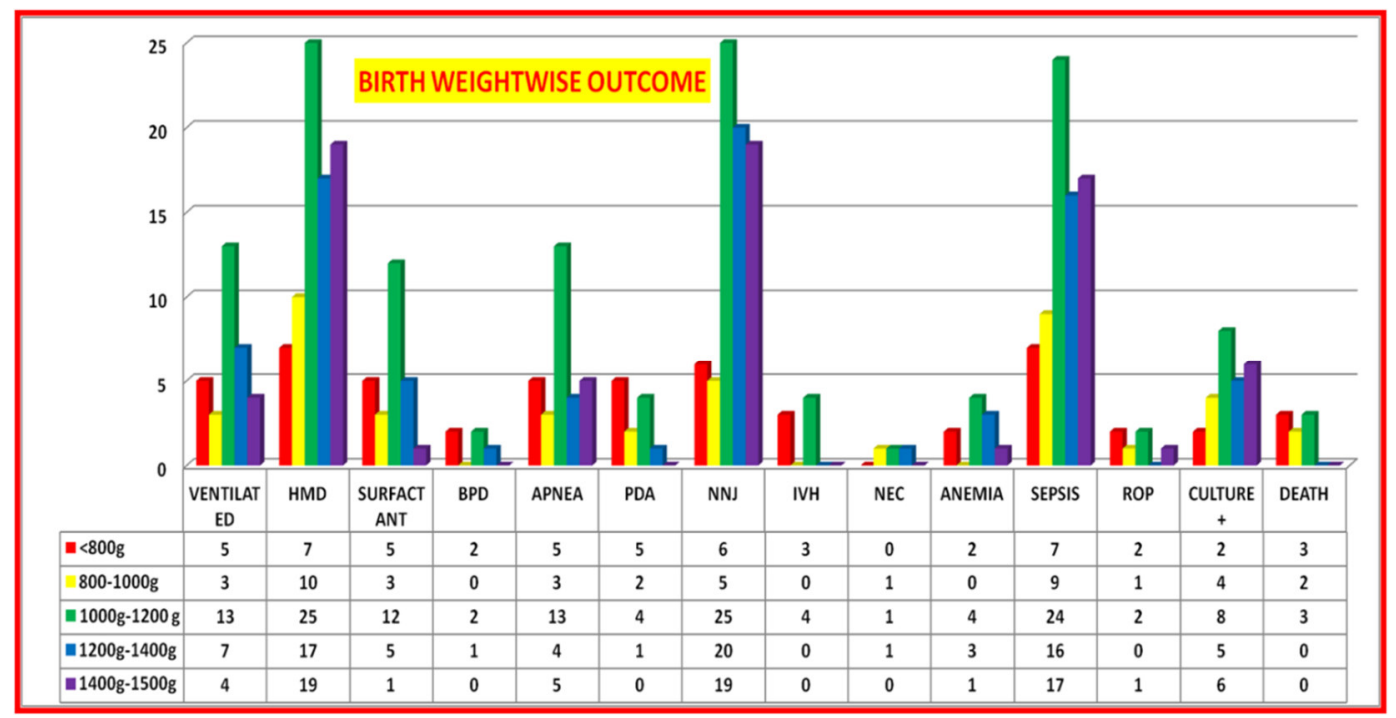

Table 5: Weight Wise Distribution of Morbidity Profile- Subgroup Analysis

\begin{tabular}{|c|c|c|c|c|c|c|c|c|c|c|c|c|}
\hline & $<0.8$ & $>0.8$ & & $<1$ & $>1$ & & $<1.2$ & $>1.2$ & & $<1.4$ & $>1.4$ & \\
\hline VENTILATED \% & 71.42 & 30 & $<0.0001$ & 47.05 & 30 & 0.0541 & 43.75 & 22.4 & 0.0087 & 38.88 & 16 & 0.0019 \\
\hline HMD \% & 100 & 78.88 & 0.1361 & 100 & 76.25 & 0.0890 & 87.5 & 73.46 & 0.2755 & 81.94 & 76 & 0.6423 \\
\hline SURFACTANT \% & 71.42 & 23.33 & $<0.0001$ & 47.05 & 22.5 & 00031 & 41.66 & 12.24 & $<0.0001$ & 34.72 & 4 & $<0.0001$ \\
\hline BPD \% & 28.57 & 3.33 & $<0.0001$ & 11.76 & 3.75 & 0.0435 & 8.33 & 2.04 & 0.2322 & 6.94 & 0 & $<0.0001$ \\
\hline APNEA $\%$ & 71.42 & 27.77 & $<0.0001$ & 47.05 & 27.5 & 0.0242 & 43.75 & 18.36 & 0.0563 & 34.72 & 20 & 0.0483 \\
\hline PDA \% & 71.42 & 7.77 & $<0.0001$ & 41.17 & 6.25 & $<0.0001$ & 22.91 & 2.04 & $<0.0001$ & 16.66 & $\mathbf{0}$ & $<0.0001$ \\
\hline NNJ $\%$ & 85.71 & 76.66 & 0.4848 & 64.70 & 71.11 & 0.5887 & 75 & 79.59 & 0.7168 & 77.77 & 76 & 0.8885 \\
\hline IVH \% & 42.85 & 4.44 & $<0.0001$ & 17.64 & 5 & 0.0079 & 14.58 & 0 & $<0.0001$ & 9.72 & 0 & $<0.0001$ \\
\hline NEC \% & $\mathbf{0}$ & 3.33 & $<0.0001$ & 5.88 & 2.5 & 0.2498 & 4.16 & 2.04 & 0.4021 & 4.16 & 0 & $<0.0001$ \\
\hline ANEMIA \% & 28.57 & 8.88 & 0.0012 & 11.76 & 10 & 0.7108 & 12.5 & 8.16 & 0.3472 & 12.5 & 4 & 0.0377 \\
\hline ROP \% & 28.57 & 4.44 & $<0.0001$ & 17.64 & 3.75 & 0.0025 & 10.41 & 2.04 & 0.0181 & 6.94 & 4 & 0.3816 \\
\hline SEPSIS \% & 100 & 73.33 & 0.0524 & 94.11 & 71.25 & 0.0784 & 83.33 & 67.34 & 0.1988 & 77.77 & 68 & 0.4259 \\
\hline CULTURE + \% & 28.57 & 25.55 & 0.6866 & 35.29 & 23.75 & 0.1379 & 29.16 & 22.44 & 0.3571 & 26.38 & 24 & 0.7418 \\
\hline DEATH \% & 42.85 & 5.55 & $<0.0001$ & 29.41 & 3.75 & $<0.0001$ & 16.66 & 0 & $<0.0001$ & 11.11 & 0 & $<0.0001$ \\
\hline
\end{tabular}

Table 5 shows the distribution of morbidity profile based upon the Birth weight subgroups.

A one-sample t-test between proportions was performed to determine whether there was a significant difference among morbidities and death based on birth weight subgroups. The t-statistic was analysed and p value $<0.05$ was considered significant. Birth weight wise $<800 \mathrm{~g} \&>800 \mathrm{~g} ;<1000 \mathrm{~g} \& 1000 \mathrm{~g} ;<1200 \mathrm{~g} \& 1200 \mathrm{~g} ;<1400 \mathrm{~g} \& 1400 \mathrm{~g}$ were analysed.

There was significant difference in Hemodynamically significant Patent Ductus Arteriosus (HS- PDA) requiring treatment and $(\mathrm{IVH})>/=$ Grade II in all subgroups. Statistical difference in need of ventilation was significant in all subgroups except between $<1 \mathrm{~kg} \&>1 \mathrm{~kg}$. Significant difference in Surfactant use, BPD was noted in all subgroups except between $<1.2 \&$ $>1.2 \mathrm{Kg}$. There was no significant difference in HMD, Culture positive and culture negative sepsis among subgroups. Significant difference in Apnea was noted between $<800 \&>800 \mathrm{~g}$. Significant difference in ROP was noted between $<800 \&$ $>800 \mathrm{~g}$ and between $<1000 \&>1000 \mathrm{~g}$. No statistical difference was noted in Hyperbilirubinemia among all subgroups. Significant difference in NEC, Anemia requiring PRBC transfusion was observed between $<800 \&>800 \mathrm{~g}$ and $<1400 \mathrm{~g} \&$ $>1400$. 
Table 6: Comparison Of Proportions / Odds Among Birth Weight Subgroups

\begin{tabular}{|c|c|c|c|c|}
\hline Morbidity & $</>800$ & $</>1000$ & $</>1200$ & $</>1400$ \\
\hline Ventilated & 2.38 & 1.56 & 1.95 & 2.43 \\
\hline HMD & 1.26 & 1.31 & 1.19 & 1.07 \\
\hline Surfactant & 3.06 & 2.09 & 3.4 & 8.68 \\
\hline BPD & 8.57 & 3.13 & 4.08 & $>$ \\
\hline Apnea & 2.57 & 1.71 & 2.38 & 1.73 \\
\hline PDA & 9.19 & 6.58 & 11.23 & $>$ \\
\hline NNJ & 1.11 & 0.9 & 0.94 & 1.02 \\
\hline IVH & 9.65 & 3.52 & $>$ & $>$ \\
\hline NEC & 0 & 2.35 & 2.03 & $>$ \\
\hline Anemia & 3.21 & 1.17 & 1.53 & 3.12 \\
\hline ROP & 6.43 & 4.7 & 5.1 & 1.73 \\
\hline sepsis & 1.36 & 1.32 & 1.23 & 1.14 \\
\hline Culture+ & 1.11 & 1.48 & 1.29 & 1.09 \\
\hline DEATH & 7.77 & 7.84 & $>$ & $>$ \\
\hline
\end{tabular}

Table 6 depicts the comparison of proportions based upon Birth Weight subgroups.

No IVH $>/=$ Grade II \& death were noted in babies with birthweight more than $1200 \mathrm{~g}$. No Hemodynamically significant Patent Ductus Arteriosus, BPD \& NEC were noted in babies with birthweight more than 1400g.Maximum difference in BPD, Apnea, Hyperbilirubinemia, IVH $>/=$ Grade II, Anemia, ROP and culture negative sepsis were noted around $800 \mathrm{~g}$ birthweight. Maximum difference in death, HMD, NEC, Culture positive sepsis were noted around 1000g. Maximum difference in Hemodynamically significant Patent Ductus Arteriosus was noted around 1200g. Maximum difference in need of surfactant and need of ventilation was noted around $1400 \mathrm{~g}$ birth weight.

\section{Discussion}

Survival rate at discharge among VLBW babies in our study was $91.75 \%$. There has been global improvement in VLBW survival. The American Academy of Pediatrics policy statement on neonatal care states that only Level III hospitals should take care for infants less than 32 weeks gestation [14]. Since 1990, Healthy People Objective for 2000, 2010 and 2020 have included the goal to increase the proportion of VLBW infants born at LEVEL III hospitals to 90 percent [15]. In 2008 the National Quality Forum endorsed a series of 17 quality measures for perinatal care. One of these quality measures states that infants $<1500 \mathrm{~g}$ should be delivered at a hospital with a Neonatal Intensive care unit[16]. Though majority of our VLBW babies were out born, we noted good survival due to prompt early referral and golden hour management including temperature. There was significant difference in need of ventilation, surfactant, Apnea, ROP, IVH $>/=$ Grade-II, Culture-negative Sepsis among all gestational subgroups in our study. Survival increased as gestation advanced. Maximum decrease in mortality occured beyond 28 weeks. Maximum odds difference in need of ventilation, BPD was noted around 28weeks. Major difference in HS-PDA, IVH, NEC occured around 30 weeks. Significant difference in need of surfactant, apnea, anemia was observed around 32weeks. Major decrease in HMD, Hyperbirubinemia, and sepsis occured around 34weeks.

J C Velaphi et al in his study found that female gender had lesser morbidity than male. Similar findings were also noted by Cartlidge et al and Stevenson DK et al $[18,19,20]$. However we did not observe the male disadvantage in our study. Birth weight in particular is strongly associated with fetal, neonatal and postneonatal mortality and with infant and child morbidity [21,22]. Impairments in fetal growth can have adverse consequences in terms of mortality, morbidity, growth and performance $[21,22,23]$.We noted Statistical significant difference in Need of ventilation, surfactant use, IVH ( $>/=$ Grade II), NEC, Anemia requiring PRBC transfusion and death between AGA and SGA. Sorina Grisaru et al in his study found that SGA infants were at increased risk for grades 3-4 ROP (OR 2.07), BPD (OR 2.52), NEC (OR 1.32) and Mortality (OR 2.37) [24]. Dhaliwal CA et al in his study found that SGA infants

were reported to be more likely to develop any stage of ROP compared to AGA peers [25]. Zaw W et al, Hallstrom $\mathrm{M}$ et al, Westby Word $\mathrm{SH}$ et al in their studies observed that SGA neonates were at increase risk for NEC $[26,27,28]$. We noted in our study that there was significant difference in Need of ventilation, surfactant use, IVH, NEC, Anemia and death was noted between AGA and SGA. While Analysing birthweight wise, 
Research Article

Survival improved as birth-weight increased in our study. There was significant difference in HS-PDA and IVH in all birth-weight subgroups. Maximum decrease in death was noted in babies $>1000 \mathrm{~g}$. Maximum odds difference in BPD, Apnea, Hyperbilirubinemia, IVH, Anemia, ROP and culture negative sepsis occured around $800 \mathrm{~g}$. Major difference in HMD, NEC, and Culture positive sepsis was observed around $1000 \mathrm{~g}$. Significant odds difference in HS-PDA occurred around $1200 \mathrm{~g}$. Maximum decrease in need of surfactant and ventilation was noted around $1400 \mathrm{~g}$.S C Velaphi et al in his study noted that the increase in survival rates by $100 \mathrm{~g}$ increments was marked between 600 and $1000 \mathrm{~g}$ compared with between 1000 and $1499 \mathrm{~g}$, reflecting severe organ immaturity at the limits of viability. He noted that the odds of survival to hospital discharge were much lower among infants weighing less than $1000 \mathrm{~g}$ and increases with increasing Birth weight, almost doubling with each increase of $100 \mathrm{~g}$ when compared with infants in the $1000-1099 \mathrm{~g}$ weight group. There were no differences in odds of survival for infants in the 1100-1199g and 1000-1099g weight groups[18].We noted Statistical significant difference in death was noted in all subgroups. Survival increased significantly as birth weight increased.

\section{Conclusion}

With advances in neonatal care particularly with VLBW and premature babies there is improvement in survival with decreasing morbidities. There was no difference between male and female in survival or morbidities. We noted improved survival with advancing Gestation, Intrauterine growth and Birth weight. Analysing intrauterine growth, significant difference in Need of ventilation, surfactant, IVH, NEC, Anemia and death was noted between AGA and SGA. Significant variations in morbidity profile were noted among different birth weight and gestational age subgroups. The present study is limited because the study population is small and the study period is short. However, a good initial database is presented and can be useful for future research in this region. If substantiated by future prospective studies, these data may help clinicians to counsel families regarding the adverse morbidity and mortality based on gestation, sex, intrauterine growth and birth weight.

Funding: Nil

Conflict of interest: Nil

Permission from IRB: Yes

Abbreviations

VLBW : Very Low Birth Weight, ROP : Retinopathy Of Prematurity, IVH : Intraventricular Hemorrhage, HS-

PDA : Hemodynamically Significant Patent Ductus
Arteriosus, NEC : Necrotising Enterocolitis , BPD: Bronchopulmonary Dysplasia, RDS : Respiratory Distress Syndrome, HMD : Hyaline Membrane Disease, PRBC : Packed Red Blood Cell, NNJ : Neonatal Jaundice, AGA : Appropriate For Gestational Age, SGA : Small For Gestational Age, NICU: Neonatal Intensive Care Unit

\section{References}

1. Ali MM, Kawser CA, Talukder. Very low birth weight infant (1500 gm or less): Clinical course in hospital. Bangladesh J Child Health 1989;13(2/3): 90-95.

2. Fanaroff AA,Hack M,Walsh MC.The NICHD neonatal research network: changes in practice and outcome during the first 15 years. Semin Perinat.2003, 27: 281-287.

3. Phibbs CS, Baker LC, Caughey AB, Danielsen B, Schmitt SK, Phibbs RH. Level and volume of neonatal intensive care and mortality of very low birth weight infants. N Engl J Medicine 2007; 356,2165-2175.

4. Phibbs CS,Bronstein JM, Buxton E, Phibbs RH, The effects of patient volume and level of care at the jospital of birth on neonatal mortality. JAMA.1996;276: 10541059.

5. Cifuentes J, Bronstein J, Phibbs CS, Phibbs RH, Schmitt SK, Carlo WA. Mortality in low birth weight infants according to level of care at hospital of birth. Pediatrics.2002;109:745-751.

6. Ruegger C, Hegglin M, Adams M,et al. Population based trends in mortality, morbidity and treatment for very preterm and very low birth weight infants over 12 years. BMC Pediatr 2012;12:17.

7. National Vital Statistics Report, National centre for health Statistics, March 31,2010;58(14): 17.

8. Ballard JL, Khoury JC, Wedig K ,Wang L, EilersWalsman BL, Lipp R. New ballard Score,expanded to include extremely premature infants. J Pediatr 1991; 119:417-23.

9. Arnall Patz. An international classification of retinopathy of prematurity. Pediatrics 1984;74-127.

10. Bancalari E, Abdenour GE, Fellar R,Ganon J . Bronchopulmonary dysplasia: J Pediatr 1979;95:819-23. 
11. Bancalari A, Abdenour G, Fellar R, Gannon J. Bronchopulmonary dysplasia: clinical presentation .J Pediatr 1979;819-22.

12. Papile I, Bursten J.Incidence and evolution of subependimal and intraventricular hemorrhage. A Study of infants with birth weights less than $1500 \mathrm{~g}$. J Pediatr 1978;92:29-34.

13. Bell MJ, Temberg JL, Feigin RD.Neonatal necrotizing enterocolitis : therapeutic decisions based upon clinical staging. Ann Surg 1978;187:1-7.

14 . Lillian Blackmon, Daniel G. Batton, Edward F. Bell, Susan E. Denson, William A. Eagle, William P. Kanto, Gilbert I. Martin, Ann R .Stark. Committee on Fetus and Newborn , Levels of Neonatal Care, Pediatrics 2004,114:1341-1347.

15. Colleen M.Ryan, Kathleen M. Turezyn, Elizabeth W.Jackson, Insum Kim, Richard J.Klein; Healthy People 2000, Review. http://www.cdc.gov/nchs/data/hp2000/hp2001.

16. Maureen P.Corry, Laura E.Riley, Magda Barini Garcia, Deborah E.Campbell, Steven L.Clark ;National Quality Forum(NQF). National Voluntary Consensus Standards for Perinatal Care 2008: A Consensus Report.Washington, DC:NQF;2009.

17. Kramer MS.Birth weight and infant mortality:perceptions and pitfalls. Paediatric and perinatal epidemiology, 1990,4:381-390.

18. S C Velaphi, M Mokhachane, R M Mphahlele, E Beckh-Amoldd, M L Kuwanda, P A Cooper; Survival of very low birth weight infants according to birth weight and gestational age in a public hospital. SAMJ, July 2005, Vol.95,No.7.

19. Cartlidge PHT, Stewart JH . Survival of very low birthweight and very preterm infants in a geographically defined population. Acta Paediatr 1997;86:105-110.

20. Stevenson D K,Verter J ,Fanaroff AA, Oh W ,Ehrenkranz RA, Shankran S ,Donovan EF, Wright LL, Lemons, Tyson JE, Korones SB, Bauer CR, Stoll B J,Papile L-A for the National Institute of Child Health and
Human Development Neonatal Research Network.Sex differences in outcome of very low birthweight infants , the newborn male disadvantage. Arch Dis Child Fetal Neonatal Ed 2000;83:F182-F185.

21. McCormick MC.The contribution of low birth weight to infant moratality and childhood morbidity. NewEngland journal of medicine,1985,312:82-90.

22. Janet C.King, Nancy F.Butte, Ronald A .Chez, Jere D.Haas,Joel C.Kleinman ;Institute of Medicine (Subcommittees on Nutritional Status and Weight Gain During Pregnancy and Dietary Intake and Nutrient Supplements During Pregnancy, Committee on Nutritional Status During Pregnancy and Lactation, Food and Nutrition Board) Nutrition During Pregnancy: Part I, Weight Gain; Part II, Nutrient Supplements. Washington, DC: National Academy Press; 1990.

23. Teberg AJ,Walther FJ, Pena IC. Mortality, morbidity and outcome of the small for gestational age infant, Seminars in perinatology,1988,12:84-94.

24. Sorina Grisaru -Granovsky ,Brian Reichman ,Liat Lerner-Geva,Vlantina Bokyo, Cathy Hammerman, ArnonSamueloff, Michael S.Schimmel; American Journal of Obstetrics \& Gynaecology, February 2012;150.e1.

25. Dhalwal CA, Fleck BW, Wright E, Graham C, Mcintosch N.Retinopathy of prematurity in small for gestational age infants compared with those of appropriate size for gestational age. Arch Dis Child 2009;94:F 193-5.

26. Zaw W, Gagnon R ,da Silva O. The risks of adverse neonatal outcome among preterm small for gestational age infants according to neonatal versus fetal growth standards . Pediatrrics 2003; 111:1273-7.

27. Haastorm M, Kolvisto AM, Janas M, Tammela O.Frequency of and risk factors for necrotizing enterocolitis in infants born before 33 weeks of gestation. Acta Pediatr 2003;92:111-3.

28. Westby Word SH, Sommerfelt K, Reigstad H. Neonatal mortality and morbidity in extremely preterm small for gestational infants: a population based study. Arch Dis Child 2009;94:F363-7. 


\section{How to cite this article?}

Rabindran, Parakh H, Ramesh J K, Reddy P. Effect of Birth Weight, Gestational Age, Sex and Intrauterine Growth on Mortality and Morbidity Profile of very Low Birth Weight Babies (VLBW). Pediatr Rev: Int J Pediatr Res 2014;1(2):40-50. doi:10.17511/ijpr.2014.i02.01 